\title{
Split energy-helicity cascades in three-dimensional homogeneous and isotropic turbulence
}

\author{
L. Biferale ${ }^{1} \uparrow$, S. Musacchio ${ }^{2}$ and F. Toschi ${ }^{3,4}$ \\ ${ }^{1}$ Department of Physics and INFN, University of Rome Tor Vergata, Via della Ricerca Scientifica 1, \\ 00133 Rome, Italy \\ ${ }^{2}$ CNRS, Laboratoire J. A. Dieudonné UMR 6621, Parc Valrose, 06108 Nice, France \\ ${ }^{3}$ Department of Physics and Department of Mathematics and Computer Science, Eindhoven University of \\ Technology, 5600 MB Eindhoven, The Netherlands \\ ${ }^{4}$ CNR-IAC, Via dei Taurini 19, 00185 Rome, Italy
}

(Received 4 March 2013; revised 21 May 2013; accepted 3 July 2013)

We investigate the transfer properties of energy and helicity fluctuations in fully developed homogeneous and isotropic turbulence by changing the nature of the nonlinear Navier-Stokes terms. We perform a surgery of all possible interactions, by keeping only those triads that have sign-definite helicity content. In order to do this, we apply an exact decomposition of the velocity field in a helical Fourier basis, as first proposed by Constantin \& Majda (Commun. Math. Phys, vol. 115, 1988, p. 435) and exploited in great detail by Waleffe (Phys. Fluids A, vol. 4, 1992, p. 350), and we evolve the Navier-Stokes dynamics keeping only those velocity components carrying a well-defined (positive or negative) helicity. The resulting dynamics preserves translational and rotational symmetries but not mirror invariance. We give clear evidence that this three-dimensional homogeneous and isotropic chiral turbulence is characterized by a stationary inverse energy cascade with a spectrum $E_{b a c k}(k) \sim k^{-5 / 3}$ and by a direct helicity cascade with a spectrum $E_{\text {forw }}(k) \sim k^{-7 / 3}$. Our results are important to highlight the dynamics and statistics of those subsets of all possible Navier-Stokes interactions responsible for reversal events in the energy-flux properties, and demonstrate that the presence of an inverse energy cascade is not necessarily connected to a two-dimensionalization of the flow. We further comment on the possible relevance of such findings to flows of geophysical interest under rotations and in thin layers. Finally we propose other innovative numerical experiments that can be achieved by using a similar decimation of degrees of freedom.

Key words: isotropic turbulence, turbulence theory

\section{Introduction}

Inviscid invariants of the NS equations are crucial to determining the direction of the turbulent energy transfer (Frisch 1995). In two-dimensional turbulence the presence of two positive definite quadratic quantities, energy and enstrophy, leads to a split 
cascade: energy flows toward large scales and enstrophy to small scales (Kraichnan 1967; Paret \& Tabeling 1998; Vorobieff, Rivera \& Ecke 1999; Clercx \& van Heijst 2009; Boffetta \& Musacchio 2010; Boffetta \& Ecke 2012; Mininni \& Pouquet 2013). The forward enstrophy cascade leads to a smooth velocity field with highly non-trivial statistical properties for the vorticity field. The backward energy cascade is close to equilibrium, with quasi-Gaussian and non-intermittent (i.e. self-similar) statistical properties. When the two-dimensional flow is forced at different scales, the two mechanisms are superimposed, as recently showed by Cencini, Muratore-Ginanneschi \& Vulpiani (2011). The fluid equations also possess two inviscid invariants in three dimensions: energy and helicity (i.e. the scalar product of velocity and vorticity). The inviscid conservation of helicity was discovered relatively recently; the first attempts to understand the phenomenology go back to the works of Moffat (1969), Brissaud et al. (1973) and Kraichnan (1973). Unlike energy, helicity is not positive definite, and therefore it is not a 'coercive' quantity. In principle, this allows a simultaneous forward transfer of energy and helicity, as confirmed by the results of two-point closures by Brissaud et al. (1973), Waleffe (1992) and André \& Lesieur (1997) and direct numerical simulations by Chen, Chen \& Eyink (2003a), Chen et al. (2003b) and Mininni \& Pouquet (2010). On the other hand, it is known that forcing mechanisms breaking the mirror symmetry may lead to inverse energy transfer at low Reynolds numbers (Sulem et al. 1989). Nevertheless, a reversal of the flux of energy has also been observed at high Reynolds numbers in geophysical flows subject to Earth rotation, as shown by Smith, Chasnov \& Waleffe (1996) and Mininni, Alexakis \& Pouquet (2009), as well as in shallow fluid layers (see e.g. Nastrom, Gage \& Jasperson 1984; Lautenschlager, Eppel \& Thacker 1998; Smith \& Waleffe 1999; Celani, Musacchio \& Vincenzi 2010; Xia et al. 2011, 2008) and in turbulent flow driven by Faraday waves (Francois et al. 2013). Recently, some hints of an inverse energy transfer have been found by Dubief, Terrapon \& Soria (2013) in a direct numerical simulation of a three-dimensional channel flow seeded with polymers. Further, quantum fluids in three dimensions may lead to an inverse transfer due to vortex reconnection (Baggaley, Barenghi \& Sergeev 2012). In rotating turbulence, energy flux is reversed only if the forcing is chiral, injecting a non-vanishing helicity in the system, while a perfect two-dimensional flow has vanishing pointwise helicity, since vorticity is always orthogonal to velocity. In both cases, energy reversal is accompanied by strong anisotropic effects and by a substantial two-dimensionalization of the flow, induced either by the rotation or by the effects of confinement. For rotating turbulence in the inverse energy cascade regime, helicity is observed to flow toward small scales: a split-cascade scenario as for the energy-enstrophy case of two-dimensional turbulence also develops in three dimensions. Similarly, the first observation of split local and non-local cascades was found in the experimental investigations of von Kármán flow at different Reynolds numbers (Herbert et al. 2012). In this work, the authors show that the energy spectrum in the direct helicity cascade changes from a slope -2 to a slope $-7 / 3$ at different Reynolds numbers. This is explained as the differing influence of local and non-local triads in the multiscale dynamics. Later in the paper we will show that for our helical Navier-Stokes equations, the spectrum is the one characterized by local interactions. The role played by helicity in the energy transfer mechanism of ideal three-dimensional isotropic flows has attracted broad scientific interest; see e.g. Chen et al. $(2003 a, b)$ and references therein. Shell models have been developed to study energy and helicity transfer at high Reynolds numbers in detail (Benzi et al. 1996; Ditlevsen 1997; Biferale 2003). Furthermore, there has been speculation concerning the existence of intermittent bursts 
in the energy cascade induced by a 'local' helicity blocking mechanism (Biferale 2003). Helicity is known to play a key role in many applied and fundamental turbulent flows with boundaries as shown by Pelz et al. (1985) and Pelz, Shtilman \& Tsinober (1986), and in conducting flows (Brandenburg 2008). Despite these important contributions, the phenomenology of helicity remains 'mysterious', as summarized in the conclusion of a state-of-the-art numerical study by Chen et al. (2003a).

In this paper we propose using an exact decomposition of the Navier-Stokes (NS) equations on a helical Fourier basis proposed originally by Constantin \& Majda (1988) (see also Waleffe 1992), in order to highlight some basic properties of helicity common to all turbulent flows. Following Waleffe (1992) we will show that the set of all triadic interactions in NS equations can be divided into four independent classes, as a function of the helical contents of each of the three interacting modes (see below for details). Our idea is to study the dynamics of NS equations by preserving only some of these classes, in order to disentangle the energy transfer as a function of the helicity properties of the interacting modes. Such experiments can be made only on computer, using numerics in its most potentially peculiar way, asking questions about the physics of NS by dividing the problem into subproblems, which cannot be done in the laboratory because degrees of freedom are always all entangled. Nevertheless, as stated in $\S 1$, there exist experimental realizations where the flow is strongly helical and where a split energy-helicity cascade scenario develops. It would be extremely interesting to perform a systematic analysis of the triadic interactions for such cases, in order to establish the relative importance of different classes of triads.

In Biferale, Musacchio \& Toschi (2012) a striking outcome has been obtained following one of the possible decimation strategies. By preserving only the class where all modes bring helicity with the same sign, the energy transfer mechanism of the resulting NS evolution is inverted with an inverse energy cascade spectrum that scales as $E_{\text {back }}(k) \sim \epsilon^{2 / 3} k^{-5 / 3}$, even in fully homogeneous and isotropic systems. This result showed that all flows in nature possess nonlinear degrees of freedom ready to transfer energy backwards, without the need to be close to a two-dimensional geometry or to be strongly anisotropic, as previously believed. Simultaneously, helicity is transferred forwards, so we are in the presence of a split-cascade scenario, as observed for flows under rotations with non-vanishing helical forcing (Mininni et al. 2009). In the presence of a direct helicity cascade, the predicted spectrum at high wavenumbers scales as $E_{\text {forw }}(k) \sim h^{2 / 3} k^{-7 / 3}$, where $h$ is the helicity flux (see Brissaud et al. 1973), leading to velocity fluctuations in real space with increments $\delta_{r} v \sim h^{1 / 3} r^{2 / 3}$, unlike the typical increment $\delta_{r} v \sim \epsilon^{1 / 3} r^{1 / 3}$ expected for the energy cascade scenario. For such a case, energy dissipation should not display any anomaly, reaching a vanishing value in the limit of infinite Reynolds numbers (Eyink \& Sreenivasan 2006). As a result, one can prove existence and uniqueness of weak solutions for these decimated NS dynamics (Biferale \& Titi 2013). The latter is the first case where this notoriously difficult problem has been solved for fully homogeneous and isotropic configurations in three dimensions.

In this paper we extend the study presented in Biferale et al. (2012) by investigating the statistical properties of the forward helicity cascade regime, i.e. the dynamics of the decimated NS (dNS) equations when forcing is restricted to low wavenumbers. Our dNS equations allow, for the first time, isolation of a forward helicity transfer without any contamination from the energy flux. Moreover, we also demonstrate the existence of two exact results generalizing the 4/5 law for the energy flux (Frisch 1995) and the 2/15 law for the helicity flux (Chkhetiani 1996), valid for the original NS case. Finally, the possible presence of intermittent corrections in the forward 
helicity cascade regime will be discussed. Let us emphasize that our numerical strategy is much more flexible and can in principle be applied to the study of different subsets of NS triads, dissecting the nonlinear terms following the dynamics in different submanifolds.

The paper is organized as follows. In $\S 2$ we briefly summarize the exact helical Fourier decomposition of the Navier-Stokes dynamics proposed by Constantin \& Majda (1988) and Waleffe (1992), and we discuss the four possible classes of triads which contribute to reconstruction of the whole nonlinear term. In $\S 3$ we summarize some of the results obtained by Biferale et al. (2012) by a direct numerical simulation of dNS equations preserving only one class of interactions, namely that one with a well-defined sign of helicity, leading to the split energy-helicity cascades. In this section we present results from numerical investigations where the forcing is at small scales, to focus on the inverse energy cascade regime. In $\S 4$ we present the main result of this paper, with novel numerical results obtained on the same subsystem but with forcing at large scales, to focus on the direct helicity cascade regime. In $\S 5$ we summarize all results, and we discuss further possible applications of our numerical methodology. In the Appendix, we present the analytical calculation needed to establish the new form of the 4/5 and 2/15 laws for the decimated NS system.

\section{Helical decomposition of Navier-Stokes dynamics}

The starting point of our analysis is the well-known helical Fourier decomposition of the velocity field $\boldsymbol{v}(\boldsymbol{x})$, expanded in Fourier components, $\boldsymbol{u}(\boldsymbol{k})$, proposed by Constantin \& Majda (1988) and Waleffe (1992). Being divergence-free, $\boldsymbol{k} \cdot \boldsymbol{u}(\boldsymbol{k})=0$, each velocity component in Fourier space has only two degrees of freedom. The idea is to define the two independent degrees of freedom by a projection onto two orthonormal helical waves with a definite sign of helicity. To do that, we define

$$
\boldsymbol{u}(\boldsymbol{k})=u^{+}(\boldsymbol{k}) \boldsymbol{h}^{+}(\boldsymbol{k})+u^{-}(\boldsymbol{k}) \boldsymbol{h}^{-}(\boldsymbol{k}),
$$

where $\boldsymbol{h}^{ \pm}$are the eigenvectors of the curl operator $\mathrm{i} \boldsymbol{k} \times \boldsymbol{h}^{ \pm}= \pm k \boldsymbol{h}^{ \pm}$. In particular, one can always choose $\boldsymbol{h}^{ \pm}=\hat{\boldsymbol{v}} \times \hat{\boldsymbol{k}} \pm \mathrm{i} \hat{\boldsymbol{v}}$, where $\hat{\boldsymbol{v}}$ is an arbitrary unit vector orthogonal to $\boldsymbol{k}$ which satisfies the relation $\hat{\boldsymbol{v}}(\boldsymbol{k})=-\hat{\boldsymbol{v}}(-\boldsymbol{k})$, necessary to ensure the reality of the velocity field (Waleffe 1992). Such a requirement is satisfied, for example, by the choice $\hat{\boldsymbol{v}}=\boldsymbol{z} \times \boldsymbol{k} /\|\boldsymbol{z} \times \boldsymbol{k}\|$, with $\boldsymbol{z}$ an arbitrary vector. In terms of this exact decomposition of each Fourier mode, the energy and the helicity,

$$
E=\int \mathrm{d}^{3} \boldsymbol{x}|\boldsymbol{v}(\boldsymbol{x})|^{2}, \quad H=\int \mathrm{d}^{3} \boldsymbol{x} \boldsymbol{v} \cdot \boldsymbol{w},
$$

where $\boldsymbol{w}$ is the vorticity, are given by the following expression:

$$
\left.\begin{array}{l}
E=\sum_{\boldsymbol{k}}\left|u^{+}(\boldsymbol{k})\right|^{2}+\left|u^{-}(\boldsymbol{k})\right|^{2}, \\
H=\sum_{\boldsymbol{k}} k\left(\left|u^{+}(\boldsymbol{k})\right|^{2}-\left|u^{-}(\boldsymbol{k})\right|^{2}\right) .
\end{array}\right\}
$$

Note that the Craya decomposition is made of two independent eigenvectors with zero helicity and it is connected to the helical decomposition via a linear transformation (Waleffe 1992). Similarly, the nonlinear term of the NS equations can be exactly decomposed into four independent classes of triadic interactions, determined by the helical content of the complex amplitudes, $u^{s_{k}}(\boldsymbol{k})$ with $s_{k}= \pm$ : see Waleffe (1992) and figure 1. Among three generic interacting modes $u^{s_{k}}(\boldsymbol{k}), u^{s_{p}}(\boldsymbol{p}), u^{s_{q}}(\boldsymbol{q})$, we can identify 


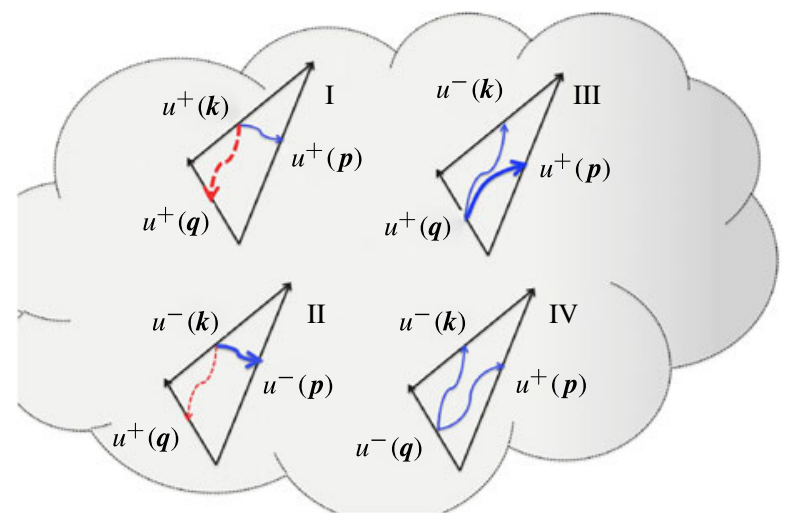

FIGURE 1. (Colour online) Helical decomposition. The triadic interaction of the NS equations are decomposed into four classes, depending on the relative helicity signs. In Waleffe (1992) a simple dynamical argument is given supporting the fact that triads of classes III and IV mainly transfer energy toward small scales (high wavenumbers), i.e. they have the usual direct cascade, whereas triads of class I enjoy an inverse energy cascade and class II is mixed. In the figure this is summarized by dashed arrows (red online) denoting a backward energy transfer and by solid arrows (blue online, arrow thickness is proportional to the energy transfer intensity) for forward energy transfer. In Biferale et al. (2012) a direct numerical integration at high resolution of the NS equations, decimated so as to keep only triads of class I, showed that a stationary turbulent inverse energy cascade is indeed established.

eight different helical combinations $\left(s_{k}= \pm, s_{p}= \pm, s_{q}= \pm\right)$. Of these, only four are independent because of the symmetry that allows us to change all signs of helicity simultaneously. The Navier-Stokes equations in the helical Fourier basis are

$$
\left(\partial_{t}+v k^{2}\right) \bar{u}^{s_{k}}(\boldsymbol{k})=-\frac{1}{4} \sum_{\boldsymbol{k}+\boldsymbol{p}+\boldsymbol{q}=0} \sum_{s_{p}, s_{q}}\left(s_{p} p-s_{q} q\right)\left[\boldsymbol{h}^{s_{p}} \times \boldsymbol{h}^{s_{q}} \cdot \boldsymbol{h}^{s_{k}}\right] u^{s_{p}}(\boldsymbol{p}) u^{s_{q}}(\boldsymbol{q}),
$$

where ${ }^{-}$stands for complex conjugate.

The core of the NS dynamics is of course in the nonlinear quadratic terms, coupling all triads. From the above expression one may extract the nonlinear dynamical evolution of each single interacting triad at three generic wavenumbers, $\boldsymbol{k}, \boldsymbol{p}, \boldsymbol{q}$ :

$$
\left.\begin{array}{l}
\bar{u}^{s_{k}}(\boldsymbol{k})=g\left(s_{p} p-s_{q} q\right) u^{s_{p}}(\boldsymbol{p}) u^{s_{q}}(\boldsymbol{q}), \\
\bar{u}^{s_{p}}(\boldsymbol{p})=g\left(s_{q} q-s_{k} k\right) u^{s_{q}}(\boldsymbol{q}) u^{s_{k}}(\boldsymbol{k}), \\
\bar{u}^{s_{q}}(\boldsymbol{q})=g\left(s_{k} k-s_{p} p\right) u^{s_{k}}(\boldsymbol{k}) u^{s_{p}}(\boldsymbol{p}),
\end{array}\right\}
$$

where $g=-1 / 4\left[\boldsymbol{h}^{s_{p}} \times \boldsymbol{h}^{s_{q}} \cdot \boldsymbol{h}^{s_{k}}\right]$ is the prefactor. It is important to note that the nonlinear dynamics preserves both energy and helicity triad by triad, i.e.

$$
\partial_{t}\left(\left|u^{s_{p}}\right|^{2}+\left|u^{s_{q}}\right|^{2}+\left|u^{s_{k}}\right|^{2}\right)=\partial_{t}\left(s_{p} p\left|u^{s_{p}}\right|^{2}+s_{q} q\left|u^{s_{q}}\right|^{2}+s_{k} k\left|u^{s_{k}}\right|^{2}\right)=0 .
$$

From a generic study of the unstable manifolds of the above nonlinear system, Waleffe (1992) showed that among the four independent helical Fourier classes of interactions there is one class that tends to transfer energy backwards (class I in figure 1), there are two classes that have mainly forward transfer (classes III and IV), and a fourth class with mixed properties (class II). Class I will be the main object of this study. It is characterized by having the same sign of helicity for all three wavenumbers in 
each triad, i.e. helicity becomes sign-definite. This is the subclass of all possible NS interactions suspected for those events of energy reversal present in all flows in nature. In order to highlight the physical, statistical and topological properties of such a class of degrees of freedom we need to propose a way to study a decimated version of the Navier-Stokes equations, projecting the dynamics onto a generic subset of all possible triads.

In order to do this, let us now consider the dynamics of an incompressible flow $\boldsymbol{\nabla} \cdot \boldsymbol{v}=0$, which is determined by a Navier-Stokes equation in which all interactions between modes have been switched off except for those with a well-defined sign of helicity, e.g. positive $\left(s_{k}=+, s_{p}=+, s_{q}=+\right)$. We define the (Galerkin) projector on positive/negative helicity states as

$$
\mathscr{P}_{i j}^{ \pm}(\boldsymbol{k}) \equiv \frac{h_{i}^{ \pm}(\boldsymbol{k}) \overline{h_{j}^{ \pm}}(\boldsymbol{k})}{\overline{\boldsymbol{h}^{ \pm}}(\boldsymbol{k}) \cdot \boldsymbol{h}^{ \pm}(\boldsymbol{k})} .
$$

The action of this projector on a field in real space must be interpreted via its Fourier decomposition. To project any field into its positive helicity component, say, we define

$$
v_{j}^{+}(\boldsymbol{x}) \equiv P_{j m}^{+} v_{m}(\boldsymbol{x}) \equiv \sum_{\boldsymbol{k}} \mathrm{e}^{\mathrm{i} \boldsymbol{k} \cdot \boldsymbol{x}} \mathscr{P}_{j m}^{+}(\boldsymbol{k}) u_{m}(\boldsymbol{k}) .
$$

We can then define the decimated NS equations with triads only of class I as

$$
\partial_{t} v_{i}^{+}=-P_{i m}^{+} \partial_{j}\left(v_{j}^{+} v_{m}^{+}\right)+v \Delta v_{i}^{+}+f_{i}^{+},
$$

where $v$ is the viscosity and $\boldsymbol{f}^{+}$is the external forcing projected onto its positive-chiral component. Let us note that pressure disappears, since each of the two components of (2.1) is already incompressible, as is also evident from (2.4). Let us note also that the projection of the nonlinear NS terms onto a definite sign of helicity introduces non-local interaction in real space. It is easy to realize that if the initial velocity configuration is chosen with only positive helicity components, $\boldsymbol{v}^{-}(\cdot, t=0)=0$, the dynamical evolution of (2.9) preserves this property for all times.

For such dynamics, since $u^{-}(\boldsymbol{k})=0$ for all $\boldsymbol{k}$, we have that helicity also becomes positive definite, as one can easily verify from (2.3). As a result, the two inviscid invariants cannot be transferred onto the same direction and we must observe a split energy-helicity transfer. In any case, let us stress that helicity becomes sign (positive) definite only when averaged on the whole system: in other words the local helicity field may still have some small regions where velocity and vorticity are not positively correlated (see also figure 3). This fact is also shown in figure 4, where we plot the probability density function of the pointwise helicity in the direct helicity cascade regime. Since helicity is more peaked at high wavenumbers, it is natural to suppose, following the equilibrium ansatz of Kraichnan (1967), that the energy flux changes sign, and the energy flows toward large scales while helicity is transferred toward small scales.

\section{Inverse energy cascade}

In Biferale et al. (2012) we have explored the dynamics of (2.9) by forcing at small scales, focusing on the inverse cascade regime. In so doing we have been able to find a clean $5 / 3$ inverse energy spectrum (see figure 5), for both unstationary and stationary statistics. In the latter case, a suitable sink of energy at small wavenumbers was introduced in order to prevent an accumulation of fluctuations in the infrared 
range (see the caption of figure 5 for more details on the DNS). Regarding the scaling properties in real space, we show in the Appendix that it is possible to generalize the exact relation known as the 4/5 law (Frisch 1995) for the third-order correlation function of the original NS case also for the dNS equation (2.9). In the dNS case, the third-order correlation function that satisfies an exact scaling relation in the limit of infinite extension of the (inverse) energy cascade is given by

$$
C_{j}(\boldsymbol{r})=-\left[\left\langle v_{i}^{+}(0) F_{i j}(\boldsymbol{r})\right\rangle-\left\langle v_{i}^{+}(\boldsymbol{r}) F_{i j}(0)\right\rangle\right],
$$

where $F_{i j}=P_{i m}^{+}\left(v_{j}^{+} v_{m}^{+}\right)$. Indeed, in the presence of a large-scale mechanism that removes the energy accumulation, one gets a stationary regime where the following exact relation holds (see (A 17) in the Appendix):

$$
C_{j}(\boldsymbol{r})=\frac{1}{3} \epsilon r_{j},
$$

where $\epsilon$ is the mean flux of energy injected by the external small-scale forcing. In figure $6(a)$ we show a confirmation of the previous relation, as it is verified in our direct numerical simulation. Notice that the small oscillations seen in the inertial range are due to the change of sign observed around the scale where the external forcing is acting, $r \sim 10 \eta$, and they are typical of all inverse cascade regimes. Let us also notice that the relation (3.1) recovers the well-known 4/5 law if one restores the complete NS dynamics, i.e. removing the helicity projector, $P_{i m}^{+}=\delta_{i m}$. The inverse cascade which arises from (2.9) is not intermittent. The probability distribution functions (p.d.f.s) of the longitudinal velocity increments $\delta_{r} v^{+}=\left[\boldsymbol{v}^{+}(\boldsymbol{x}+\boldsymbol{r})-\boldsymbol{v}^{+}(\boldsymbol{x})\right] \cdot \hat{\boldsymbol{r}}$ at distance $r$ within the inertial range are self-similar and almost Gaussian (see figure $6 b$ ). The scaling of the second- and the fourth-order moments of velocity increments,

$$
S_{2}(r)=\left\langle\left(\delta_{r} v+\right)^{2}\right\rangle, \quad S_{4}(r)=\left\langle\left(\delta_{r} v^{+}\right)^{4}\right\rangle,
$$

follow the dimensional scaling $S_{p}(r) \sim r^{p / 3}$ : see Biferale et al. (2012). This is a peculiarity of all inverse cascades observed so far (see e.g. Boffetta \& Ecke 2012 for the case of two-dimensional NS), i.e. whenever the cascade proceeds from faster to slower degrees of freedom, as already observed in dynamical models by Benzi, Biferale \& Sbragaglia (2005).

\section{Direct helicity cascade}

The simultaneous conservation of energy and helicity, which is responsible for the reversal of the energy cascade, must lead to the development of a direct helicity cascade at scales smaller than the correlation scale of the forcing.

In order to study the range of scales where the physics is dominated by the direct helicity cascade, it is necessary to make a different set of simulations in which the flow is forced at small wavenumbers. The new set of simulations has been performed with a fully dealiased, pseudospectral code at resolution $512^{3}$ on a triply periodic cubic domain of size $L=2 \pi$. The flow is sustained by a random Gaussian forcing, with $\left\langle f_{i}(\boldsymbol{k}, t) f_{j}\left(\boldsymbol{q}, t^{\prime}\right)\right\rangle=F(k) \delta(\boldsymbol{k}-\boldsymbol{q}) \delta\left(t-t^{\prime}\right) Q_{i j}(\boldsymbol{k})$, where $Q_{i j}(\boldsymbol{k})$ is a projector ensuring incompressibility and $F(k)$ has support only in the low-wavenumber range $|k| \in[1: 3]$. In the absence of a large-scale dissipative mechanism, the inverse cascade would accumulate the kinetic energy in the lowest available mode, leading to the formation of a condensed state (Xia et al. 2011). In order to reduce this phenomenon we added hypo-viscosity at large scales $\propto \Delta^{-1} v$. In such a case, the total kinetic energy becomes stationary, as shown in figure 7 , and it is equally distributed among the three velocity components, showing that the flow is statistically isotropic. 

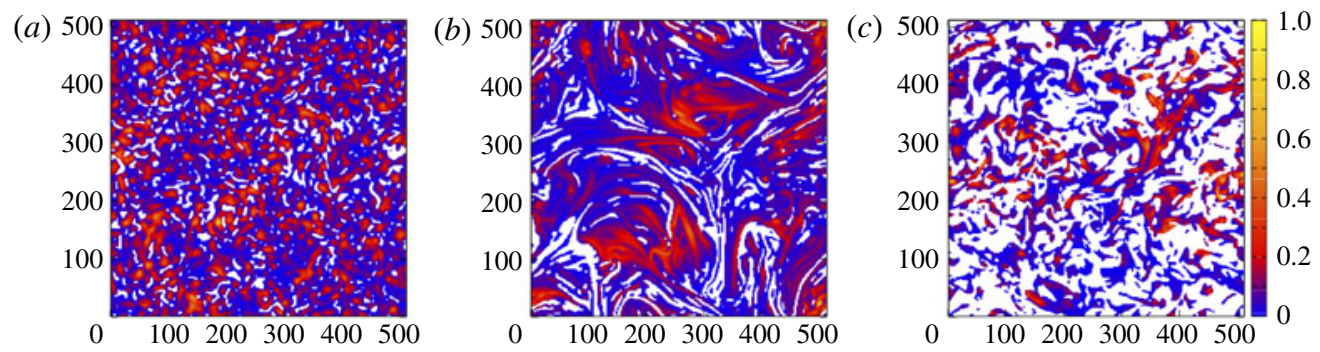

FIgURE 2. (Colour online) Two-dimensional snapshot of the local helicity field $\boldsymbol{v}(\boldsymbol{x}) \cdot \boldsymbol{w}(\boldsymbol{x})$ for three different simulations: $(a)$ decimated Navier-Stokes with small-scale forcing (inverse energy cascade regime); $(b)$ decimated Navier-Stokes with large-scale forcing (direct helicity cascade regime); (c) original undecimated Navier-Stokes with homogeneous, isotropic and parity-invariant forcing. Note that to better highlight the asymmetry between positive and negative helicity values we plot only positive values of helicity (white spots correspond to negative values).
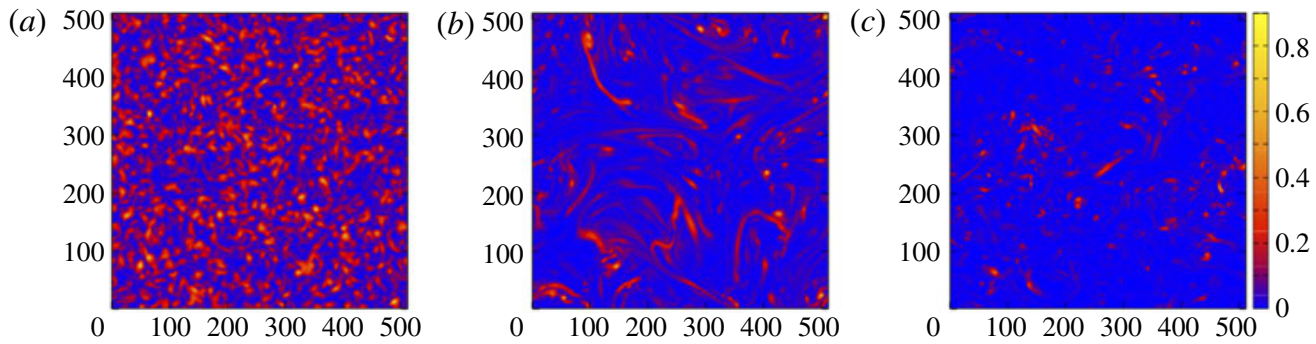

FIgURE 3. (Colour online) The same as figure 2 but for the total vorticity amplitude $w(x) \cdot w(x)$.

This allows us to study scaling properties without having to deal with anisotropic secondary contributions, in contrast to what happens for rotating turbulence (Biferale \& Procaccia 2005; Mininni et al. 2009). Let us first make a general phenomenological comment. As one can see in figures 2-3, the spatial fluctuations of helicity and vorticity are very different depending on whether one performs numerical experiments with small-scale or large-scale forcing, and they are also very different from the typical realizations observed in a fully homogeneous, isotropic and parity-invariant undecimated Navier-Stokes system. For instance, figure 2 clearly shows the formation of coherent large-scale helical structures for the case of large-scale forcing in positive definite Navier-Stokes (figure $2 b$ ), which are almost absent in the inverse energy cascade regimes and completely absent in the classical NS parity-invariant case, where spatial correlations are weak and perfect symmetry between positive and negative helicity values is observed. A different scenario develops when comparing the amplitude of the vorticity field (figure 3). Now we observe that the formation of coherent structures is present only in figure $3(a)$, corresponding to the usual 


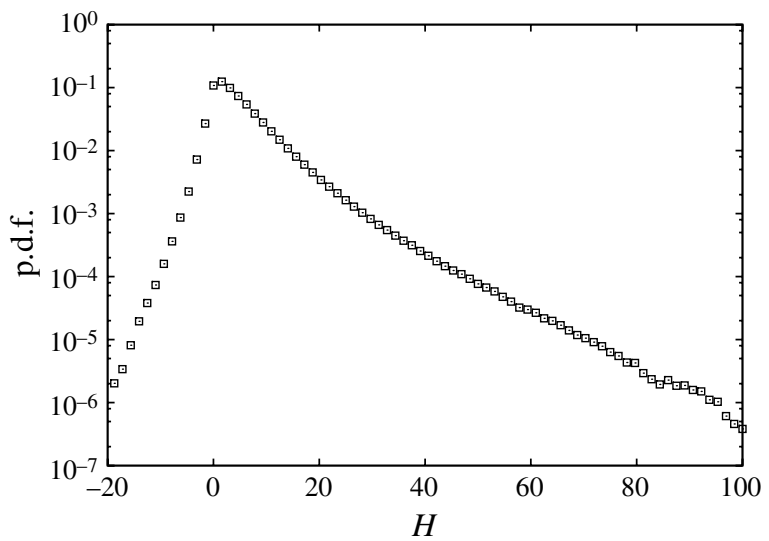

FIGURE 4. Probability density function of the pointwise helicity, $\boldsymbol{w}(\boldsymbol{x}) \cdot \boldsymbol{v}(\boldsymbol{x})$, in the real space for the decimated Navier-Stokes case with helicity positive definite in Fourier space. Note the existence of localized events in real space with negative values.
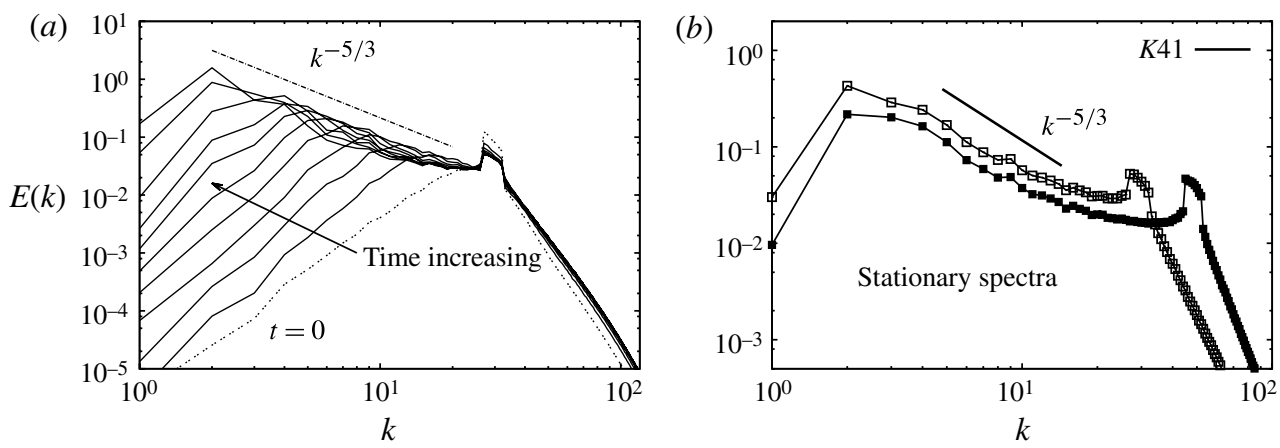

FIGURE 5. Results from a DNS of the dNS equation (2.9) at $512^{3}$ collocation points using a fully dealiased pseudospectral method. (a) Spectrum obtained from a non-stationary evolution (no sink of energy at large scales), starting with energy at $t=0$ only at high wavenumbers (dashed line). Forcing is random and delta-correlated in time acting on $k_{f} \in[28: 35]$. (b) Two spectra obtained with forcing acting in two different windows, $k_{f} \in[28: 35]$ and $k_{f} \in$ [42:50], with large-scale removal of energy to obtain stationary statistics at two effective Reynolds numbers. The energy removal at large scales is obtained adding an inverse Laplacian, $\propto k^{-\beta} \boldsymbol{u}^{+}(\boldsymbol{k})$, in the evolution of the $\boldsymbol{k}$ Fourier velocity component.

homogeneous and isotropic parity-invariant flows, whereas when helicity dominates, the dynamic structures are less coherent and eventually present only on a much larger scale (figure $3 b$ ), as it must be in the helical decimated NS because of the absence of a strong vortex-stretching mechanism. In figure 8 we show the energy spectrum obtained from (2.9) by initializing the flow with energy only at low wavenumbers. The development of a direct cascade with a spectrum $E(k) \sim k^{-7 / 3}$ is unambiguous. Evidence of direct helicity cascades has already been reported in fluid under rotation and with a helical input at the forcing scale (Mininni 2011). In that case, the presence of strong anisotropy and the limitations in the scale resolution did not allow to go beyond a qualitative statement. Similarly, a direct helicity cascade with a spectrum 

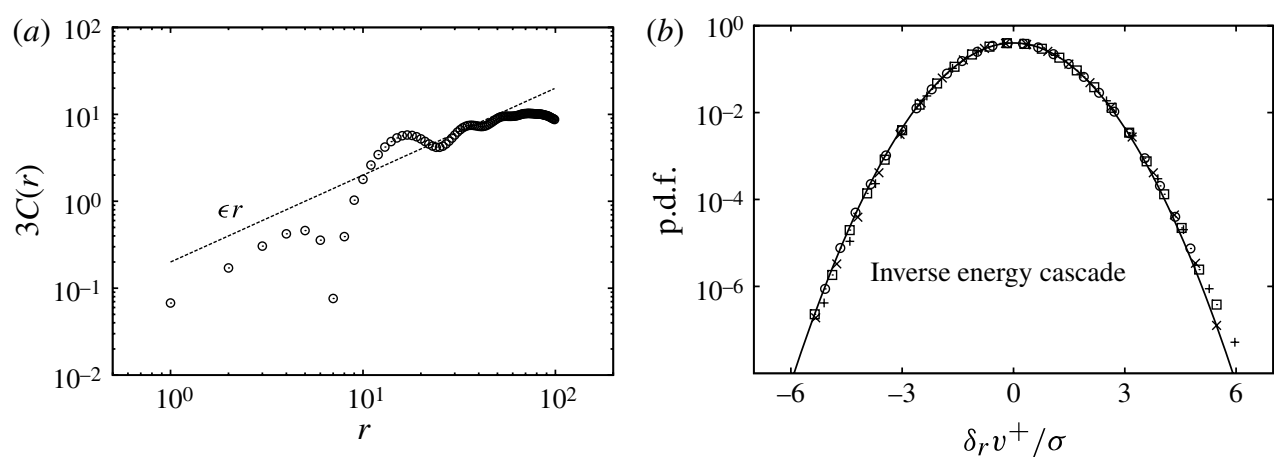

FIGURE 6. Inverse energy cascade range. (a) Exact third-order relation in the inverse cascade regime. Here we plot the longitudinal component $3 C_{j}(r) \hat{r}_{j}$ versus $r$ in units of the Kolmogorov scale $\eta$. The straight line corresponds to the prediction (3.2). (b) Probability density function for velocity increments normalized with their standard deviation, $\sigma_{r}$, for three different $r / \eta$ values inside the inertial range. The solid line is the Gaussian p.d.f.

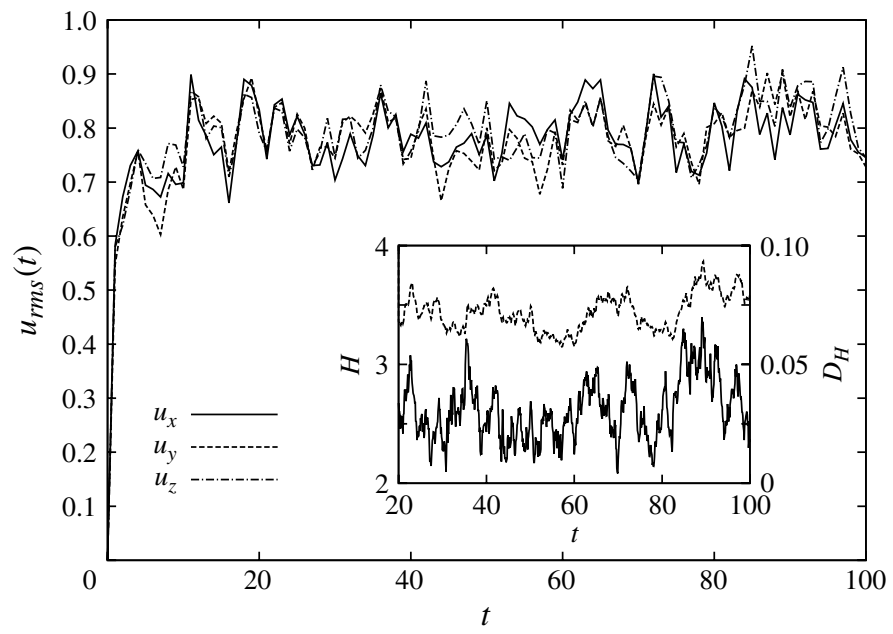

FIGURE 7. The evolution of the three $u_{r m s}$ components show that in the steady state the flow is isotropic. The inset shows the time evolution of the total helicity (solid line) and the helicity dissipation (dashed line). Note the small delay between the peaks of the two quantities typical of all forward cascade regimes.

slope changing between -2 and $-7 / 3$, depending on the Reynolds number, has been observed in an experimental von Kármán flow by Herbert et al. (2012), the two limits being connected to the cases of non-local or local cascades.

In figure $8(b)$ we show the stationary spectrum obtained in our simulation compensated with the slopes $-7 / 3$ and -2 . The first is in perfect agreement with our data, indicating that the main helicity transfer occurs via local interactions in Fourier space. In contrast, the latter is not consistent with our results. To our knowledge this is the first time that a clear $-7 / 3$ spectrum due to a helicity cascade has been observed in turbulence. 

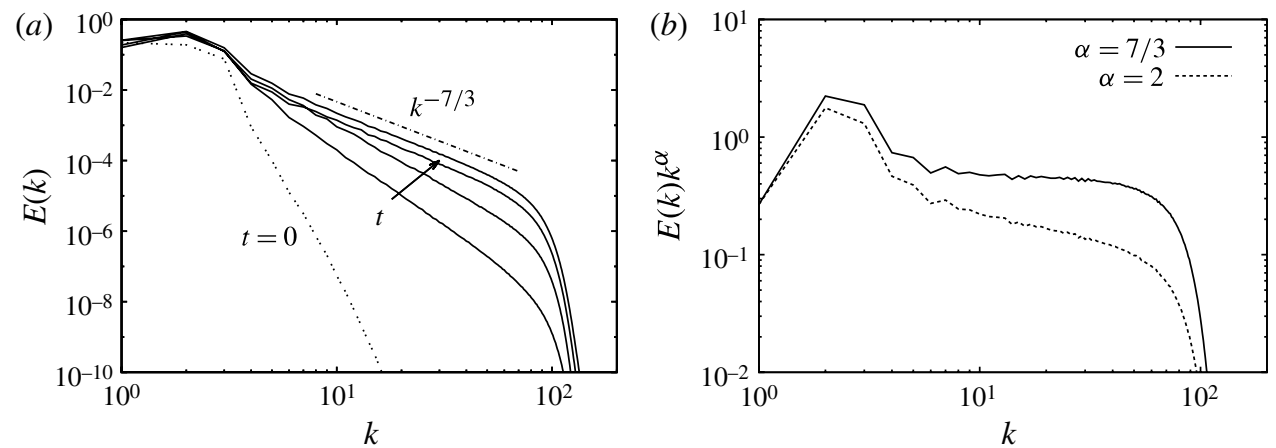

FIGURE 8. Results from a DNS of (2.9) with a large-scale forcing (see the text for details). (a) The energy spectra at various times show the development of the direct helicity cascade. At $t=0$ the velocity fields contain energy only at large scales. In the steady state $(t>20)$ a spectrum $E(k) \sim k^{-7 / 3}$ is observed. $(b)$ The spectrum in the steady state compensated with $k^{-7 / 3}$ and $k^{-2}$.

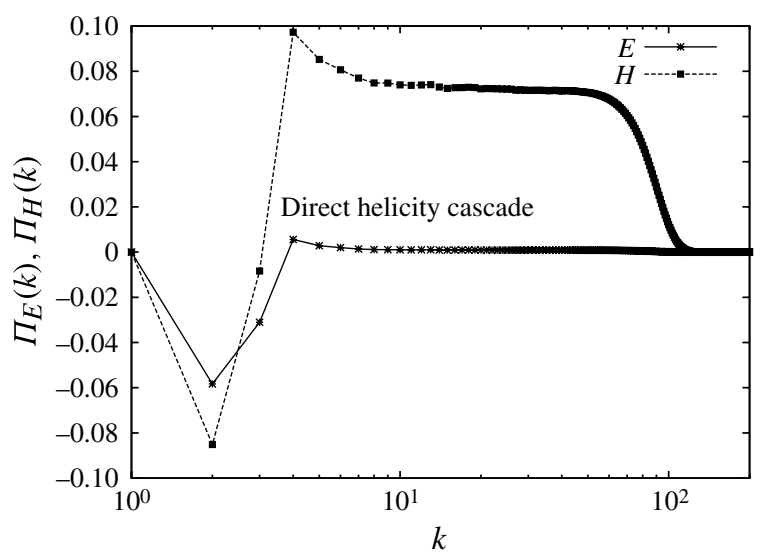

FIGURE 9. Results from a DNS of (2.9) with a large-scale forcing (see the text for details).

Helicity (top curve) and energy (bottom curve) flux in Fourier space $\Pi_{H}(k), \Pi_{E}(k)$.

In figure 9 we show the stationary helicity flux in Fourier space, defined as $\Pi_{H}(k) \equiv(\mathrm{d} / \mathrm{d} t) \int_{k}^{\infty} H(p) \mathrm{d} p$, where the time derivative is computed by taking into account only the nonlinear terms of (2.9). The plateau at a positive value for wavenumbers in the inertial range is a clear indication of the helicity transfer toward small scales.

The presence of a constant non-zero flux of helicity allows us to derive a second exact relation for homogeneous, isotropic, non-parity-invariant turbulence. In particular, assuming stationarity, it is possible to show that the tensor

$$
H_{j l}(\boldsymbol{r})=\epsilon_{i j k}\left[\left\langle F_{k l}^{\prime} v_{i}\right\rangle-\left\langle v_{k}^{\prime} F_{i l}\right\rangle\right]
$$

has the following expression (see the Appendix):

$$
H_{j l}(\boldsymbol{r})=\left(a+b r^{2}\right) \delta_{j l}+c r^{2} \hat{r}_{j} \hat{r}_{l},
$$




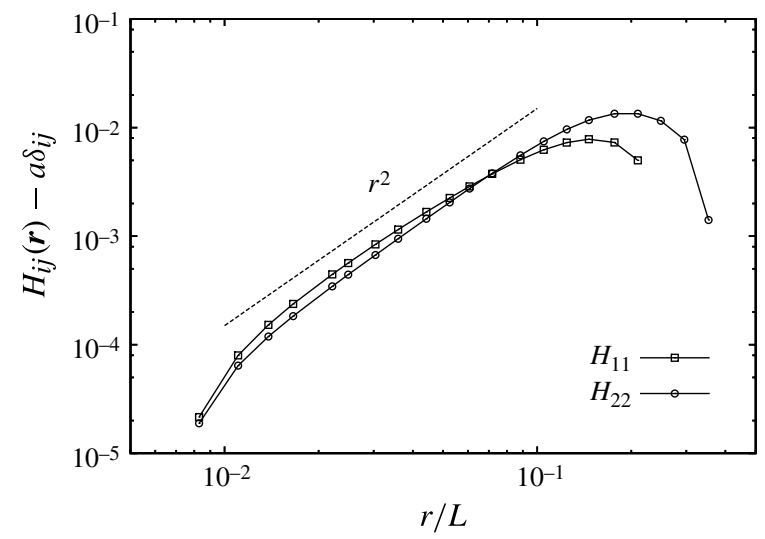

FIGURE 10. Scaling behaviour of two components of the tensor $H_{j l}(\mathbf{r})$. The scaling $r^{2}$ is represented by the dashed line.

with the constraint $6 b+12 c=h$, where $h$ is the helicity input. In figure 10 we show $H_{11}$ and $H_{22}$ for the case $\hat{r}=(1,0,0)$, in which the tensor $H_{j l}(\boldsymbol{r})$ is diagonal. Let us notice that, given the isotropy of the flow, the direction of $\hat{r}$ is immaterial and $H_{22}=H_{33}$. In each case, the $r^{2}$ behaviour in the scaling range is observed. An estimate of the coefficients $a, b$ from $H_{22}$ allows us to obtain a prediction for $H_{11}$ without free parameters, which is in close agreement with the data.

\subsection{Small-scale statistics}

Unlike inverse cascades, direct turbulent cascades are often observed to break selfsimilarity and are characterized by anomalous scaling laws accompanied by strong intermittent small-scale fluctuations. Here, the quantity which is transferred toward small scales is the helicity, and not energy. The resulting velocity field is much smoother than in the usual case of a direct energy cascade. Indeed, the energy spectrum $E(k) \sim k^{-7 / 3}$ observed in our numerics is consistent with the dimensional scaling in real space for velocity increments in the inertial range, i.e.

$$
\delta_{r} v \sim h^{1 / 3} r^{2 / 3},
$$

where $h$ denotes the intensity of the helicity flux. In order to study whether the forward helicity cascade breaks the self-similar scaling (4.3) we need to measure different moments of velocity increments (structure functions), as already done in the inverse energy cascade regime. Unfortunately, a roughness exponent $\sim 2 / 3$ is very close to the differentiable value $\sim 1$, which is also the limit where non-local effects start to play a key role between Fourier modes. In such a situation, we expect that the scaling in real space can be strongly contaminated by the accumulation of energy at small wavenumbers, due to the inverse energy cascade. In spite of the use of a large-scale dissipation to reduce this phenomenon, such accumulation is clearly visible in the spectrum obtained in our simulation. Indeed the structure functions of order $p=2,4,6$ display a strong contamination at all scales due to the infrared part of the spectrum, and do not match the dimensional scaling (see figure 11). Let us note that by performing an ad hoc filtering of fluctuations in the low-wavenumber regimes one may strongly reduce the importance of the large-scale energy accumulation, obtaining a scaling closer to the $2 / 3$ exponent for second-order structure functions (not shown). 

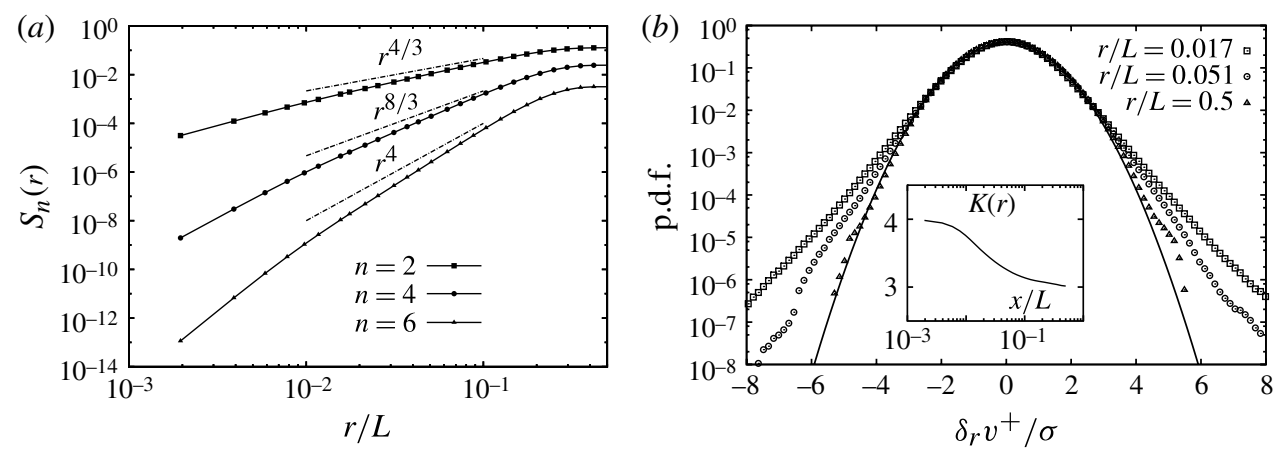

FIGURE 11. (a) Longitudinal velocity structure functions of order $n=2,4,6$. Dash-dotted lines represent the dimensional scaling $S_{n}(r) \sim r^{2 n / 3}$. (b) Probability density functions of longitudinal velocity increments normalized with their variance for various separations $r$. The inset shows the kurtosis $K(r)$.

In figure 11 we also show the p.d.f.s of longitudinal velocity increments rescaled to have unit variance for different separations in the inertial and viscous range. It is evident that there is not a perfect rescaling. At large separations the p.d.f.s are close to Gaussian, but their tails become wider and wider by decreasing the separation $r$. This is a clear qualitative indication that the forward helicity cascade is intermittent. In the inset of the same figure we also show the behaviour of the kurtosis,

$$
K(r)=\frac{S_{4}(r)}{\left(S_{2}(r)\right)^{2}},
$$

which provides a quantitative indicator of deviations from Gaussianity (for a Gaussian distribution we have $K(r)=3$ ). Again, the growth of $K(r)$ by decreasing the scale $r$ is a quantitative measure of intermittency. More numerical simulations are needed, in any case, to make a firm assessment of the universality of such anomalous fluctuations, due to the presence of the huge energy accumulations at low wavenumbers shown by the spectrum. Such a bump may well be so intense as to contaminate the inertial range, leading to spurious (i.e. Reynolds-dependent) intermittent corrections. Other analysing tools which have proved to be optimal to reduce non-local effects, such as the Hilbert-Huang transform (Huang \& Shen 2005), may also help to better clarify this point. If intermittency in the helical cascade is confirmed, it will be important to study it in a more quantitative way in order to understand similarities to and differences from the corresponding phenomenon observed for the whole NS dynamics with all triads included and in the presence of rotation (Mininni et al. 2009). This may shed some light on the nature of anomalous scaling in turbulence and its dependence on the physical properties of the nonlinear degrees of freedom.

\section{Conclusions}

We have investigated the transfer properties of the energy and helicity fluctuations in fully developed homogeneous and isotropic turbulence where the nature of the nonlinear terms of the Navier-Stokes equations have been modified ad hoc. This careful surgery of the nonlinear terms allows us to retain, among all possible triadic interactions, only those that preserve the sign of the helicity. The theoretical procedure is demonstrated on a set of decimated NS equations, that are numerically solved 
by means of a projection on the helical Fourier basis, first proposed by Waleffe (1992), and by a pseudospectral solver. We demonstrate that the resulting dynamics preserves homogeneity and isotropy, and we give clear evidence of the development of a stationary inverse energy cascade and of a direct helicity cascade. Our findings clearly indicate that among all possible interactions, in three-dimensional turbulence there is always a subset of the triadic coupling that naturally produces an inverse energy cascade. Our findings might also help to rationalize the emergence and transition between two- and three-dimensional turbulence phenomenology in flows where rotation and/or geometrical confinement enforces constraints on the helicity. In particular, it is worth noting that Xia et al. (2011) have shown that the presence of a mean rotating flow can enhance the inverse energy cascade in fluid layers. This result seems to suggest that mean flows can play a selective role in triadic interactions. Many other possible studies can be performed following this route. For example, one natural interesting development consists in performing the opposite surgery, removing only those triads with the same helicity components. In the latter case, a direct energy cascade is thought to develop, but with unknown statistical properties. Whatever the result, i.e. whether the small-scale fluctuations are the same as or different to the original undecimated NS case, it might be of key importance to unravel the universal properties of three-dimensional Navier-Stokes turbulent flows. The result of novel numerical simulation pertaining to this last case will be reported elsewhere. Let us also note that very similar decompositions in (magnetic) helical waves can be performed for conducting flows in the Coulomb gauge, i.e. with a divergencefree magnetic potential (Lessiness, Plunian \& Carati 2009). In such a case too, one may hope to learn about the entangled dynamics of the three inviscid invariants, total energy, magnetic helicity and velocity-magnetic cross-correlation, by performing suitable surgeries in subspaces.

Finally, we would like to stress that other decimation strategies might offer alternative potential insight into the phenomenology of turbulence. For example, for approaches based on Galerkin projections, we cite the idea of keeping modes in Fourier space corresponding to wavenumbers logarithmically equispaced (Grossmann et al. 1996), or on a Fractal set (Frisch et al. 2012). Similarly, Laval, Dubrulle \& Nazarenko (2001) proposed multiscale triad pruning, in order to distinguish the role played by local and non-local Fourier interactions in the growth of intermittency.

\section{Acknowledgements}

We acknowledge useful discussion with U. Frisch. L.B. acknowledges the kind hospitality of the Observatoire de la Côte d'Azur in Nice, where part of this work was done. We acknowledge the European COST Action MP0806.

\section{Appendix}

A.1. Von Kármán-Howarth equation for decimated NS equations in the inverse energy cascade regime

Let us start with the dNS equations written as in (2.9):

$$
\partial_{t} v_{i}^{+}=P_{i m} \partial_{j}\left(v_{m}^{+} v_{j}^{+}\right)+v \Delta v_{i}^{+}+f_{i}^{+},
$$

where the pressure disappears because the projector projects onto a zero-divergence field, $\mathscr{P}_{j m}^{+}(k) k_{m}=0$ for all $k_{m}$. 
Notice that projection and the derivative commute. Indeed,

$$
\partial_{j} v_{m}^{+}(\boldsymbol{x}) v_{j}^{+}(\boldsymbol{x})=\sum_{\boldsymbol{q}} \mathrm{i} q_{j}\left[\sum_{\boldsymbol{p}+\boldsymbol{k}=\boldsymbol{q}} u_{m}^{+}(\boldsymbol{p}) u_{j}^{+}(\boldsymbol{k})\right] \exp (\mathrm{i} \boldsymbol{q} \cdot \boldsymbol{x})
$$

and

$$
P_{i m}^{+}\left[\partial_{j} v_{m}^{+}(\boldsymbol{x}) v_{j}^{+}(\boldsymbol{x})\right]=\sum_{\boldsymbol{q}} \mathrm{i} q_{j} \mathscr{P}_{m j}^{+}(\boldsymbol{q})\left[\sum_{p+k=q} u_{m}^{+}(\boldsymbol{p}) u_{j}^{+}(\boldsymbol{k})\right] \exp (\mathrm{i} \boldsymbol{q} \cdot \boldsymbol{x}) .
$$

Similarly we have

$$
\begin{aligned}
\partial_{j} P_{i m}^{+}\left(\partial_{j} v_{m}^{+}(\boldsymbol{x}) v_{j}^{+}(\boldsymbol{x})\right) & =\partial_{j} \sum_{q} \mathscr{P}_{m j}^{+}(\boldsymbol{q})\left[\sum_{\boldsymbol{p}+\boldsymbol{k}=\boldsymbol{q}} u_{m}^{+}(\boldsymbol{p}) u_{j}^{+}(\boldsymbol{k})\right] \exp (\mathrm{i} \boldsymbol{q} \cdot \boldsymbol{x}) \\
& =\sum_{q} \mathrm{i} q_{j} \mathscr{P}_{m j}^{+}(\boldsymbol{q})\left[\sum_{\boldsymbol{p}+\boldsymbol{k}=q} u_{m}^{+}(\boldsymbol{p}) u_{j}^{+}(\boldsymbol{k})\right] \exp (\mathrm{i} \boldsymbol{q} \cdot \boldsymbol{x}) .
\end{aligned}
$$

So, introducing $F_{i j}=P_{i m}^{+}\left(v_{m}^{+} v_{j}^{+}\right)$, we can write

$$
\partial_{t} v_{i}^{+}=-\partial_{j} P_{i m}^{+}\left(v_{m}^{+} v_{j}^{+}\right)=-\partial_{j} F_{i j} .
$$

Let us continue and look at the nonlinear terms first, following the notation of Frisch (1995). We want to control the evolution of the two-point correlation functions $\left\langle v_{i}^{\prime} v_{i}\right\rangle$, where we use $v$ to denote the fields in $\boldsymbol{x}$ and $v^{\prime}$ in $\boldsymbol{x}^{\prime}$. Moreover, we write $\boldsymbol{r}=\boldsymbol{x}^{\prime}-\boldsymbol{x}$. Of course, we have for homogeneity $\partial_{r_{i}}=\partial_{i}^{\prime}=-\partial_{i}$.

For simplicity of notation, let us write $v_{i}^{+}=v_{i}$, so that

$$
\partial_{t}\left\langle v_{i} v_{l}^{\prime}\right\rangle=-\left\langle v_{i} \partial_{j}^{\prime} F_{l j}^{\prime}\right\rangle-\left\langle v_{l}^{\prime} \partial_{j} F_{i j}\right\rangle
$$

Using homogeneity, (A 7) can be written as

$$
\partial_{t}\left\langle v_{i} v_{l}^{\prime}\right\rangle=-\partial_{r_{j}}\left[\left\langle v_{i} F_{l j}^{\prime}\right\rangle-\left\langle v_{l}^{\prime} F_{i j}\right\rangle\right] .
$$

The third-order tensors $\left\langle v_{i} F_{l j}^{\prime}\right\rangle$ and $\left\langle v_{l}^{\prime} F_{i j}\right\rangle$ are no longer symmetric for the indexes $l j$ or $i j$. If we write

$$
b_{i j m}(\boldsymbol{r})=\left\langle v_{i} F_{j m}^{\prime}\right\rangle,
$$

then the general expression (assuming homogeneity and isotropy but not parity invariance) is

$$
\begin{aligned}
b_{i j m}(\boldsymbol{r})= & A(r) \delta_{i j} \hat{r}_{m}+B(r) \delta_{i m} \hat{r}_{j}+C(r) \delta_{j m} \hat{r}_{i}+D(r) \hat{r}_{i} \hat{r}_{j} \hat{r}_{m} \\
& +E(r) \epsilon_{i k m} \hat{r}_{k} \hat{r}_{j}+F(r) \epsilon_{i k m j} \hat{r}_{k} \hat{r}_{m}+G(r) \epsilon_{j k m} \hat{r}_{k} \hat{r}_{i}+M(r) \epsilon_{i j m} .
\end{aligned}
$$

The above expression has eight unknowns and incompressibility will add only three constraints. So the general third-order correlation function without symmetries on the indexes is not closed in terms of only one function (in contrast to what happens when it has two indexes that are symmetric, i.e. for the usual NS equations). Nevertheless, we can derive a sort of 4/5 law when we look at the trace of the two-point correlation function (i.e. the equation for the energy); see also Falkovich, Fouxon \& Oz (2010). Let us also put back forcing and viscosity:

$$
\partial_{t}\left\langle v_{i} v_{i}^{\prime}\right\rangle=-\partial_{r_{j}}\left[\left\langle v_{i} F_{i j}^{\prime}\right\rangle-\left\langle v_{i}^{\prime} F_{i j}\right\rangle\right]+\left\langle f_{i} v_{i}^{\prime}\right\rangle+\left\langle f_{i}^{\prime} v_{i}\right\rangle+2 v \partial_{r_{j}, r_{j}}\left\langle v_{i} v_{i}^{\prime}\right\rangle .
$$


To derive the equivalent for the 4/5 law as for non-decimated NS in two or three dimensions we need to add some input: we assume either a direct energy cascade or an inverse one. We will work in the latter case, looking at scales larger than the typical scale of the forcing. Suppose we have an external forcing with a typical correlation on the scale $l_{f}$, and we consider the behaviour of (A 12) for $r=\left|\boldsymbol{x}^{\prime}-\boldsymbol{x}\right| \gg l_{f}$. Then

$$
\left\langle f_{i} v_{i}^{\prime}\right\rangle+\left\langle f_{i}^{\prime} v_{i}\right\rangle \sim f_{0} \exp \left(-\left|\boldsymbol{x}^{\prime}-\boldsymbol{x}\right| / l_{f}\right) \sim 0, \quad r \gg l_{f} .
$$

Further, conservation of energy implies

$$
\partial_{t}\left(v_{i}\right)^{2}=2\left\langle v_{i} f_{i}\right\rangle=\epsilon,
$$

where the viscous term is negligible in the inverse energy cascade regime. Going back to (A 12), we see that for $\left|\boldsymbol{x}-\boldsymbol{x}^{\prime}\right| \gg l_{f}$, again neglecting the viscous contribution, we obtain

$$
\partial_{r_{j}} C_{j}(\boldsymbol{r})=\epsilon,
$$

where $C_{j}(\boldsymbol{r})=-\left[\left\langle v_{i} F_{i j}^{\prime}\right\rangle-\left\langle v_{i}^{\prime} F_{i j}\right\rangle\right]$. Here we have taken the leading term $\partial_{t}\left\langle v^{\prime} v\right\rangle \sim$ $\partial_{t}\left\langle v^{2}\right\rangle=\epsilon$, which is correct once the velocity field develops an inverse energy cascade with a power law slope $\sim r^{1 / 3}$.

If it is not parity-invariant, the mean value of a vector can only be written as

$$
C_{j}(\boldsymbol{r})=A(r) \hat{r}_{j} .
$$

The only solution to (A 15) is again

$$
C_{j}(\boldsymbol{r})=\frac{1}{3} \epsilon r_{j},
$$

i.e. we have the equivalent of the $4 / 5$ law for the third-order quantity:

$$
\left[\left\langle v_{i} F_{i j}^{\prime}\right\rangle-\left\langle v_{i}^{\prime} F_{i j}\right\rangle\right]=\frac{1}{3} \epsilon r_{j} .
$$

A similar result would have been obtained adding a large-scale friction term in (A 12) in order to get a stationary inverse cascade, exactly like the inverse cascade in two dimensions.

\section{A.2. Von Kármán-Howarth equation for decimated NS equations in the direct helicity cascade regime}

For the scaling of third-order quantities connected to the presence of a net helicity flux, we need to look at the time evolution of second-order correlation functions also involving the vorticity (Chkhetiani 1996; Biferale, Pierotti \& Toschi 1998). Starting from the dNS (A 6), where we write for simplicity only the inertial terms,

$$
\partial_{t} v_{i}=-\partial_{j} P_{i m}\left(v_{m} v_{j}\right)=-\partial_{j} F_{i j},
$$

we have for vorticity

$$
\partial_{t} w_{i}=-\epsilon_{i j k} \partial_{j} \partial_{l} F_{k l} .
$$

So for the two-point correlation

$$
\partial_{t}\left\langle v_{i} w_{i}^{\prime}\right\rangle=-\partial_{r_{j}} \partial_{r_{l}} \epsilon_{i j k}\left[\left\langle F_{k l}^{\prime} v_{i}\right\rangle-\left\langle v_{k}^{\prime} F_{i l}\right\rangle\right]+h,
$$

where $h$ is the input of helicity from the forcing. Now, the tensor

$$
c_{i l k}(\boldsymbol{r})=\left\langle F_{k l}^{\prime} v_{i}\right\rangle-\left\langle v_{k}^{\prime} F_{i l}\right\rangle
$$


also has the most general expression as in (A 11). To obtain a close exact scaling relation, it is sufficient to make a contraction with the fully anti-symmetric tensor, to obtain

$$
H_{j l}(\boldsymbol{r})=\epsilon_{i j k}\left[\left\langle F_{k l}^{\prime} v_{i}\right\rangle-\left\langle v_{k}^{\prime} F_{i l}\right\rangle\right],
$$

and to notice that the von Kármán-Howarth equation (A 21), assuming stationarity, is

$$
\partial_{r_{j}} \partial_{r_{l}} H_{j l}=h \text {. }
$$

Moreover, because $H$ is contracted with a symmetric tensor $\partial_{l} \partial_{j}$, the only part that contributes is

$$
H_{j l}(\boldsymbol{r})=h_{1}(r) \delta_{j l}+h_{2}(r) \hat{r}_{j} \hat{r}_{l}
$$

and it is easy to see that (A 24) admits a solution of the form

$$
h_{1}(r)=a+b r^{2}, \quad h_{2}(r)=c r^{2},
$$

where $a, b$ and $c$ are proportional to the helicity input $h$ and satisfy the constraint $2 d b+d(1+d) c=h$, where $d$ is the spatial dimension.

In conclusion we have

$$
H_{i j}(\boldsymbol{r})-H_{i j}(0)=r^{2}\left(b \delta_{i j}+c \hat{r}_{j} \hat{r}_{i}\right)
$$

\section{REFERENCES}

ANDRÉ, J. C. \& LESIEUR, M. 1977 Influence of helicity on the evolution of isotropic turbulence at high Reynolds number. J. Fluid Mech. 81, 187-207.

Baggaley, A. W., Barenghi, C. F. \& Sergeev, Y. A. 2012 Three-dimensional inverse energy cascade induced by vortex reconnections. arXiv:1208.5204.

Benzi, R., Biferale, L., KerR, R. \& Trovatore, E. 1996 Helical shell models for three-dimensional turbulence. Phys. Rev. E 53, 3541-3550.

Benzi, R., Biferale, L. \& Sbragaglia, M. 2005 Dynamical scaling and intermittency in shell models of turbulence. Phys. Rev. E 71, 065302.

Biferale, L. 2003 Shell models of energy cascade in turbulence. Annu. Rev. Fluid Mech. 35, 441-468.

Biferale, L., Musacchio, S. \& Toschi, F. 2012 Inverse energy cascade in three-dimensional isotropic turbulence. Phys. Rev. Lett. 108, 164501.

Biferale, L., Pierotti, D. \& Toschi, F. 1998 Helicity transfer in turbulent models. Phys. Rev. E 57, R2515-R2518.

Biferale, L. \& Procaccia, I. 2005 Anisotropy in turbulent flows and in turbulent transport. Phys. Rep. 414, 43-164.

Biferale, L. \& Titi, E. 2013 On the global regularity of a helical-decimated version of the 3D Navier-Stokes equations. J. Stat. Phys. 151, 1089-1098.

Boffetta, G. \& Ecke, R. E. 2012 Two-dimensional turbulence. Annu. Rev. Fluid Mech. 44, 427-451.

Boffetta, G. \& Musacchio, S. 2010 Evidence for the double cascade scenario in twodimensional turbulence. Phys. Rev. E 82, 016307.

BRANDEnburG, A. 2008 The inverse cascade and nonlinear alpha-effect in simulations of isotropic helical hydromagnetic turbulence. Astrophys. J. 550, 824-840.

Brissaud, A., Frisch, U., Leorat, J., Lesieur, M. \& Mazure, M. 1973 Helicity cascades in fully developed isotropic turbulence. Phys. Fluids 16, 1366-1367.

Celani, A., Musacchio, S. \& Vincenzi, D. 2010 Turbulence in more than two and less than three dimensions. Phys. Rev. Lett. 104, 184506. 
Cencini, M., Muratore-Ginanneschi, P. \& Vulpiani, A. 2011 Nonlinear superposition of direct and inverse cascades in two-dimensional turbulence forced at large and small scales. Phys. Rev. Lett. 107, 174502.

Chen, Q., Chen, S. \& EYINK, G. L. $2003 a$ The joint cascade of energy and helicity in three-dimensional turbulence. Phys. Fluids 15, 361-374.

Chen, Q., Chen, S., Eyink, G. L \& Holm, D. D. $2003 b$ Intermittency in the joint cascade of energy and helicity. Phys. Rev. Lett. 90, 214503.

Chrhetiani, O. G. 1996 On the third moments in helical turbulence. J. Expl Theor. Phys. Lett. 63, $808-812$.

Clercx, H. J. H. \& VAn Heijst, G. J. F. 2009 Two-dimensional Navier-Stokes turbulence in bounded domains. Appl. Mech. Rev. 62, 020802.

Constantin, P. \& MAJdA, A. 1988 The Beltrami spectrum for incompressible fluid flows. Commun. Math. Phys. 115, 435-456.

Ditlevsen, P. D. 1997 Cascades of energy and helicity in the GOY shell model of turbulence. Phys. Fluids 9, 1482-1484.

Dubief, Y., Terrapon, V. E. \& Soria, J. 2013 On the mechanism of elasto-inertial turbulence. arXiv:1301.3952v1.

Eyink, G. L. \& SREenivasAn, K. R. 2006 Onsager and the theory of hydrodynamic turbulence. Rev. Mod. Phys. 78, 87-135.

FAlkovich, G., Fouxon, I. \& Oz, Y. 2010 New relations for correlation functions in Navier-Stokes turbulence. J. Fluid Mech. 644, 465-472.

Francois, N., Xia, H., Punzmann, H. \& Shats, M. 2013 Inverse energy cascade and emergence of large-scale coherent vortices in turbulence driven by Faraday waves. arXiv:1302.2993.

FRISCH, U. 1995 Turbulence: The Legacy of A. N. Kolmogorov. Cambridge University Press.

Frisch, U., Pomyalov, A., Procaccia, I. \& RaY, S. S. 2012 Turbulence in non-integer dimensions by fractal Fourier decimation. Phys. Rev. Lett. 108, 074501.

Grossmann, S., Lohse, D \& ReEH, A. 1996 Developed turbulence: from full simulations to full mode reductions. Phys. Rev. Lett. 77, 5369-5372.

Herbert, E., Daviaud, F., Dubrulle, B., Nazarenko, S. \& Naso, A. 2012 Dual non-Kolmogorov cascades in a von Kármán flow. Europhys. Lett. 100, 44003.

HuAng, N. E. \& SHEN, S. S. 2005 Hilbert-Huang Transform and its Applications. World Scientific.

Kraichnan, R. H. 1967 Inertial ranges in two-dimensional turbulence. Phys. Fluids 10, 1417-1423.

Kraichnan, R. H. 1973 Helical turbulence and absolute equilibrium. J. Fluid Mech. 59, 745-752.

Lautenschlager, M., Eppel, D. P. \& Thacker, W. C. 1998 Subgrid parametrization in helical flows. Beitr. Phys. Atmos. 61, 87-97.

Laval, J.-P., Dubrulle, B. \& Nazarenko, S. 2001 Nonlocality and intermittency in three-dimensional turbulence. Phys. Fluids 13, 1995-2012.

Lessinnes, T., Plunian, F. \& Carati, D. 2009 Helical shell models for MHD. Theor. Comput. Fluid Dyn. 23, 439-450.

MininNi, P. D. 2011 Scale interactions in magnetohydrodynamic turbulence. Annu. Rev. Fluid Mech. 43, 377-397.

Mininni, P. D., Alexakis, A. \& Pouquet, A. 2009 Scale interactions and scaling laws in rotating flows at moderate Rossby numbers and large Reynolds numbers. Phys. Fluids 21, 015108 .

Mininni, P. D. \& Pouquet, A. 2010 Rotating helical turbulence. Part 2. Intermittency, scale invariance, and structures. Phys. Fluids 22, 035105.

Mininni, P. D. \& PouQuet, A. 2013 Inverse cascade behaviour in freely decaying two-dimensional fluid turbulence. arXiv:1302.2988.

Moffat, H. K. 1969 The degree of knottedness of tangled vortex lines. J. Fluid Mech. 35, $117-129$.

Nastrom, S. G., Gage, K. S. \& Jasperson, W. H. 1984 Kinetic energy spectrum of large- and mesoscale atmospheric processes. Nature 310, 36-38. 
PARET, J. \& TABeling, P. 1998 Intermittency in the two-dimensional inverse cascade of energy: experimental observations. Phys. Fluids 10, 3126-3136.

Pelz, R. B., Shtilman, L. \& Tsinober, A. 1986 The helical nature of unforced turbulent flows. Phys. Fluids 29, 3506-3508.

Pelz, R. B., Yakhot, V., Orszag, S. A., Shtilman, L. \& Levich, E. 1985 Velocity-vorticity patterns in turbulent flow. Phys. Rev. Lett. 54, 2505-2508.

Smith, L. M., Chasnov, J. R. \& Waleffe, F. 1996 Crossover from two- to three-dimensional turbulence. Phys. Rev. Lett. 77, 2467-2470.

SMith, L. M. \& WALEFFE, F. 1999 Transfer of energy to two-dimensional large scales in forced, rotating three-dimensional turbulence. Phys. Fluids 11, 1608-1622.

Sulem, P. L., She, Z. S., SChOll, H. \& Frisch, U. 1989 Generation of large-scale structures in three-dimensional flow lacking parity-invariance. J. Fluid Mech. 205, 341-358.

Vorobieff, P., Rivera, M. \& ECKE, R. E. 1999 Soap film flows: statistics of two-dimensional turbulence. Phys. Fluids 11, 2167-2177.

WALEFFE, F. 1992 The nature of triad interactions in homogeneous turbulence. Phys. Fluids A 4, 350-363.

Xia, H., Byrne, D., Falkovich, G. \& Shats, M. G. 2011 Upscale energy transfer in thick turbulent fluid layers. Nature Phys. 7, 321-324.

Xia, H., Punzmann, H., Falkovich, G. \& Shats, M. G. 2008 Turbulence-condensate interaction in two dimensions. Phys. Rev. Lett. 101, 194504. 